\title{
Estereolitografía en Odontología: Revisión bibliográfica
}

\section{Artículo de ReVisión}

\author{
Stereolitography in Dentistry: Literature review
}

\begin{abstract}
Resumen
Uno de los sistemas de prototipado más utilizado en odontología es la estereolitograff́a. El uso eficiente de la estereolitografía en Odontología ya está bien consolidado y demostrado en la práctica y en la literatura. Posee múltiples aplicaciones especialmente en las especialidades de ortodoncia, implantología, cirugía maxilofacial, traumatología maxilofacial y prótesis maxilofacial. A pesar de que la maquinaria en la técnica de estereolitografía presenta un alto costo, esta tecnología está cada vez más presente en la práctica odontológica como recurso auxiliar para casos complejos.

Palabras clave: Ortodoncia, Cirugía Maxilofacia; CAD-CAM; Procedimientos quirúrgicos orales.
\end{abstract}

\section{Abstract}

Stereolithography is the most common prototyping system used in dentistry, the efficient use of Stereolithography in dentistry is well documented in literature and in practice. It has multiple aplications, especially in orthodontics, implantology, maxillofacial surgery and maxillofacial traumatology. Despite the high cost of the machines used in this technique, each day this technology is more present in the daily practice of dentists, as an auxiliary resource for complex cases.

Keywords: Orthodontics; Oral Surgery; CAD-CAM; Oral surgical procedures

\author{
Noemí Leiva ${ }^{1, a, b}$, Francisca Carranza ${ }^{1, a}$, \\ Ignacia Sat ${ }^{1, a}$
}

\author{
1 Facultad de Odontología. Universidad de Chi- \\ le. Santiago, Chile. \\ a Cirujano Dentista \\ b Especialista de Ortodoncia y Ortopedia Maxi- \\ lofacial
}

Correspondencia:

Noemí Leiva

Correo electrónico: leivanoemi@yahoo.com

Calle Sergio Roberto Livingstone Pohlhammer 943 Comuna de Independencia.

Santiago de Chile, Chile

\section{Coautores:}

Francisca Carranza

facarranza@miuandes.cl

Ignacia Sat

ignaciasat@gmail.com

Conflicto de intereses: Los autores declaran no tener conflictos de interés.

Fuente de financiamiento: Autofinanciado.

Fecha de recepción: 17/05/17

Fecha de aceptación: 29/06/17

\section{Introducción}

El término prototipado rápido (PR) se refiere a un conjunto de tecnologías que se basan en la construcción de estructuras físicas tridimensionales, corte por corte, a partir de datos computacionales. Es un término genérico utilizado para definir las tecnologías que pueden fabricar, de manera rápida, objetos físicos directamente de fuentes de datos del sistema computacional CAD (Computer Aided Design). Esta técnica de construcción de objetos sólidos parte del corte en secciones horizontales paralelas de piezas representadas en el CAD. Estas secciones construyen las formas sólidas a partir de la superposición de capas horizontales ${ }^{1}$.

La fuente de datos de la imagen 3D es generalmente proveniente de la TC, aunque la Resonancia Magnética y la
Ultrasonografía pueden ser también imágenes utilizadas, siempre y cuando los datos estén en formato DICOM ( $D i$ gital Imaging and Communication in $\mathrm{Me}$ dicine). El proceso que ocurre durante la transferencia de datos del CAD para el equipo de PR se muestra en la figura 1 .

Inicialmente, los datos DICOM son procesados en el sistema CAD, representado por el paso 1. En una próxima etapa, paso 2, el modelo es convertido en un formato denominado STL (Standard Tercekation Language). En el paso 3, se lleva a cabo el proceso de fabricación del modelo, en donde los planos horizontales intersectan el modelo creado en el sistema CAD. Después de esto, toda la información es transferida para el equipo de prototipo rápido para ser utilizado. Dependiendo de la tecnología, el paso 4 es realizado para un post procesamiento de prototipo, como acabado, lijado o pintado. Todo el ciclo del proceso puede ser repetido cuantas veces sea necesario ${ }^{1}$.

Las aplicaciones iniciales debido a su costo fueron la ingeniería aeroespacial, industrias de automóviles y para organismos gubernamentales; sin embargo, hoy día presenta numerosas aplicaciones como las que encontramos en ingeniería, diseño, medicina, paleontología, odontología $^{2}$.

Así mismo comenzó a ser utilizado en el área biomédica; en Odontología, se usa principalmente en la fabricación de modelos para planificación quirúrgica, implantología, ortodoncia, cirugía máxilofacial y prótesis maxilofaciales ${ }^{1}$.

Las técnicas de prototipado rápido más utilizadas son la Estereolitografía (SL), la Sinterización Selectiva por Laser (SLS), la Impresión Tridimensional 
(3D Printing), el Modelaje por Deposición Fundida (FDM) y el Thermojet. Todas estas se basan en el principio de adición por capas del material, que corresponden a los "cortes" axiales de la estructura anatómica examinada ${ }^{3}$.

El sistema de prototipado más utilizado en la odontología es la Estereolitografia (SL, Stereolithography), donde los modelos tridimensionales son construidos a partir de polímeros líquidos sensibles a la luz, que se solidifican cuando son expuestos a la radiación ultravioleta. Esta solidificación se va realizando por capas hasta completar la pieza ${ }^{3}$. La figura 2 muestra un esquema de máquina de estereolitografía.

La Sinterización Selectiva a Láser (SLS, Selective Laser Sintering), es una técnica en la cual se usa un rayo láser para fundir, de forma selectiva, materiales como nailon, elastómeros y metales, en un objeto sólido. El Modelaje por deposición de material fundido (FDM - Fused Deposition Modeling), corresponde a una técnica donde los modelos son confeccionados a partir de la deposición de filamentos de resina termoplástica calentada. La Impresión Tridimensional (3DP - 3D Printing), produce modelos mediante la aposición de capas a través de la aglutinación de yeso y almidón ${ }^{1}$. La figura 3 muestra un esquema de máquina de sinterización por láser.

El objetivo del siguiente artículo es presentar una revisión de la técnica de estereolitografía, dirigido a sus principales aplicaciones en ortodoncia y cirugía máxilofacial, resaltando las ventajas, desventajas y limitaciones.

\section{Aplicaciones de Estereolitografía en Odontología}

La estereolitografía, en sus aplicaciones médicas u odontológicas, se realiza a partir de archivos DICOM, generados en estudios imagenológicos tales como

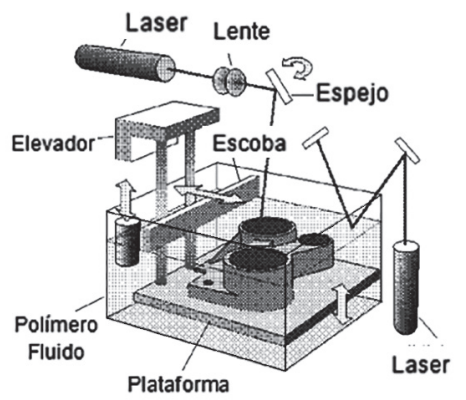

Figura 2. Esquema del equipamiento de estereolitografía ${ }^{1}$.

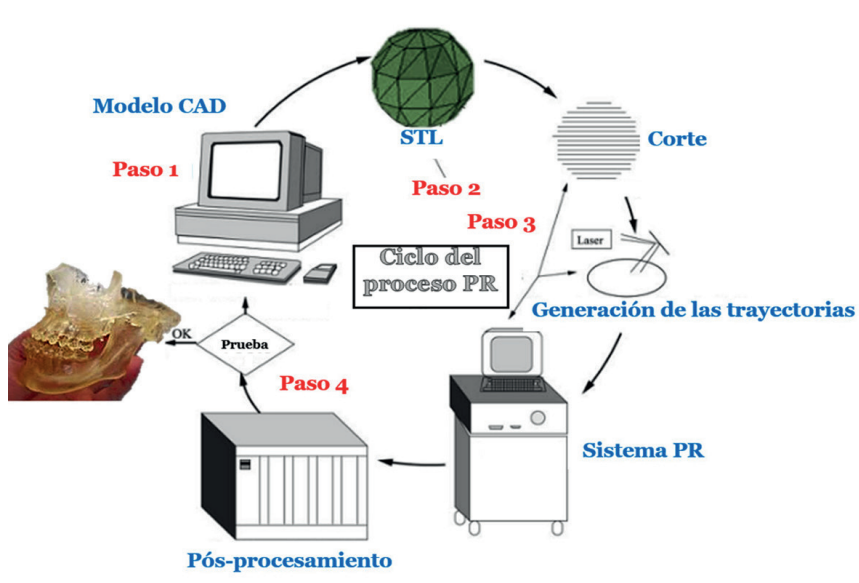

Figura 1. Proceso de Prototipado. Rápido ${ }^{1}$.

TAC (tomografía axial computada), RMI (resonancia magnética por imagen), ecografía y CBTC (comunications based trained control). Estos datos son interpretados por un software que segmenta la información y la transforma en archivos stl y la envía a una impresora. Con toda esta información correctamente procesada se puede generar, mediante la estereolitografía, un modelo en tres dimensiones de tamaño real y sólido. En la figura 4, se observan modelos obtenidos mediante la técnica de estereolitografía ${ }^{2}$.

El uso eficiente de la estereolitografía en odontología ya está bien consolidado y demostrado en la literatura ${ }^{1,4-13}$. El prototipado rápido posee muchas aplicaciones en la odontología especialmente en las especialidades de ortodoncia, implantología y, principalmente, en la cirugía maxilofacial y traumatología buco maxilofacial ${ }^{4}$.

\section{Estereolitografía y Cirugía Maxilofacial}

Anderl y cols. en 1994 determinaron que los biomodelos ayudan en la visualización de estructuras que se utilizan en el estudio de ciertas deformidades

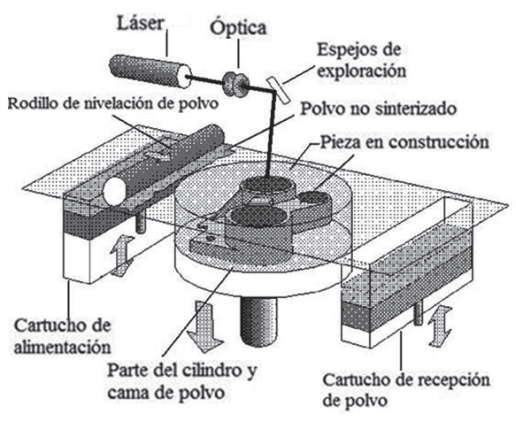

Figura 3. Esquema de máquina de Sinterización por láser ${ }^{1}$. faciales, reduciendo el riesgo quirúrgico, además de optimizar el planeamiento de osteotomías y del movimiento de fragmentos óseos 5 .

Sailer y cols. en 1998 afirmaron que los modelos obtenidos por estereolitografía sirven para la simulación de osteotomías, medición de estructuras, entrenamiento de técnicas de resección y una completa planificación de los diversos tipos de cirugía de la región buco maxilofacial ${ }^{6}$.

Según Salles y cols. en el 2002 los modelos confeccionados por la técnica de estereolitografía permiten la percepción táctil de la anatomía de la región y patología en estudio, lo cual permite la simulación y planificación quirúrgica. En relación a la simulación del procedimiento, existe una ventaja de disminución de cerca del 30\% del tiempo operatorio $^{7}$.

En el área de cirugía maxilofacial, Mazzonetto y cols. en el 2002, determinaron que la obtención de biomodelos permite un diagnóstico más preciso y,
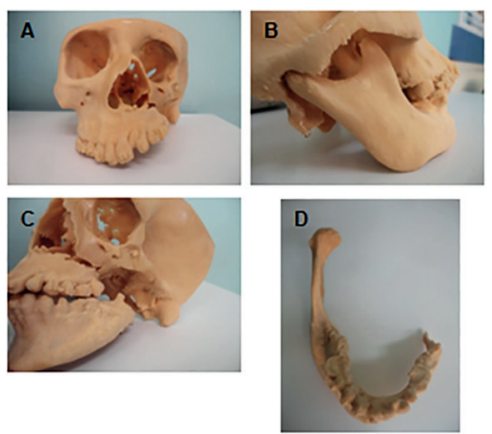

Figura 4. Imágenes de modelos confeccionados a través de estereolitografía de paciente Microsomía Hemifacial tipo III. A. Fotografía vista frontal. B. Fotografía vista lateral izquierda. C. Fotografía $3 / 4$ vista lateral derecha. D. Fotografía vista superior de mandíbula. 
consecuentemente, una mejor planificación quirúrgica en cirugías reconstructivas, cirugías ortognáticas, cirugías de ATM, en el tratamiento de las lesiones de naturaleza traumática y distracciones osteogénicas ${ }^{1,4,7,8}$.

Cunningham y cols. en el 2005 obtuvieron éxito en la utilización de los biomodelos en la planificación de cirugías reconstructivas de mandíbula y en la definición de las dimensiones de injerto óseo en pacientes sometidos a la resección de tumores en la región ${ }^{8}$.

En relación a la disminución del tiempo preoperatorio, Toro y cols. en el 2007, realizaron un estudio en donde se encontró una disminución de $1-1,5$ horas al momento de utilizar modelos realizados mediante estereolitografía, durante las cirugías de cinco casos clínicos; en comparación con la realización del mismo tipo de cirugía donde no se utilizaron este tipo de modelos 9 .

Por otro lado, en el estudio realizado por Zinser y cols. se compararon los tiempos operatorios utilizando tres métodos de confección de férulas, entre ellos el método tradicional y el uso de impresión 3D. Los resultados indican que el tiempo promedio que dura una cirugía utilizando férulas confeccionadas tradicionalmente es de 4,3 horas. Utilizando las férulas CAD/CAM se aumentó en 20 min el tiempo de la cirugía, debido a la preparación que requería del campo quirúrgico ${ }^{10}$.

\section{Estereolitografía y Ortodoncia}

En ortodoncia, la estereolitografía desempeña un papel significativo en la confección de modelos para colocación de micro tornillos ortodóncicos y la coordinación ortodoncista - cirujano. Estos dispositivos son útiles para alcanzar un anclaje absoluto durante el movimiento dentario. Según los resultados obtenidos en el estudio de Morea y cols., en el 2011, los micro tornillos instalados guiándose con el método de la estereolitografía, presentarían mayor beneficio en aquellos pacientes con múltiples piezas impactadas o con limitada distancia interradicular, donde la precisión de la instalación fue mayor con este método de prototipado rápido ${ }^{11}$.

Otra aplicación en el área es la colaboración de la estereolitografía con los modelos de estudio de yeso. Según el estudio realizado por Hazeveld en el 2014, los modelos obtenidos mediante la técnica de estereolitografía fueron considerados clínicamente aceptables en términos de precisión y reproductibilidad; por lo tanto pueden ser utilizados para ciertas aplicaciones en ortodoncia ${ }^{12}$.

En el área de diagnóstico ortodóncico, Cuperus y cols. determinaron la validez y reproductibilidad de las medidas dentarias y segmentos de arcos dentarios en modelos obtenidos por estereolitografía y por scanner intraoral. Se estableció que los modelos obtenidos por ambos métodos son válidos y reproducibles para medir distancias en una dentición ${ }^{13-18}$.

Faber y cols. utilizaron modelos realizados con estereolitogafía como una herramienta para realizar diagnósticos ortodóncicos y planificación de tratamiento de caninos superiores impactados. Las imágenes de los caninos impactados obtenidas por tomografía computarizada fueron importadas a un procesador de prototipado, para finalmente ser enviados a la impresora 3D donde son construídos los modelos en resina acrílica polimerizada por luz UV. Los modelos mostraron la relación anatómica exacta entre las piezas impactadas y las otras piezas dentarias, sirviendo como guía durante la cirugía. El modelo también fue utilizado como herramienta para comunicarse con los padres del paciente y para la fabricación de los aditamentos para la tracción del canino ${ }^{19}$.

La lengua entrega un papel importante en el crecimiento facial. Salles y cols. describen el reporte de un caso clínico de un paciente con aglosia que presentaba una alteración dentofacial que afectaba la mandíbula en particular. Los modelos obtenidos por prototipado rápido fueron usados para la fabricación de un distractor para realizar distracción osteogénica de la sínfisis mandibular ${ }^{20}$.

\section{Ventajas y Limitaciones de la Estereolitografía en Odontología}

La estereolitografía se ha convertido en uno de los métodos de modelos utilizables más populares debido a la precisión en los resultados obtenidos, destacando como principal ventaja la excelente calidad de superficie. Por estos motivos, ha sido este método el más utilizado en la odontología, específicamente en ortodoncia y cirugía maxilofacial, en comparación con los otros métodos de prototipado rápido (Tabla 1).

En las especialidades de ortodoncia y cirugía maxilofacial la estereolitografía presenta múltiples aplicaciones (Tabla 2).

La estereolitografía corresponde a la técnica pionera en odontología, ya que posee un buen acabado y ausencia de contaminación por partículas; pero por otro lado, tiene la desventaja que requiere mucho tiempo para producir el producto final ${ }^{1}$.

Tabla 1. Comparación de las diferentes técnicas de prototipado rápido ${ }^{2}$

\begin{tabular}{|c|c|c|c|c|}
\hline Propiedades & Estereolitografía & $\begin{array}{c}\text { Sinterización selectiva por } \\
\text { Laser }\end{array}$ & $\begin{array}{l}\text { Modelaje por deposición } \\
\text { Fundida }\end{array}$ & Impresión tridimensional \\
\hline Materiales & Resina fotopolimerizable & Compuestos de nylon, cerámica y cera. & Policarbonatos & Resinas líquidas con fotopolímeros \\
\hline Precisión & Excelente & Suficiente & Pobre & Buena \\
\hline Estabilidad dimensional & Buena & Excelente & Buena & Buena \\
\hline Rápido tiempo de fabricación & Suficiente & Buena & Excelente & Suficiente \\
\hline Reproducción de formas complejas & Excelente & Suficiente & Suficiente & Pobre \\
\hline Acabado superficial & Bueno & Suficiente & Pobre & Excelente \\
\hline Componentes con múltiples materiales & No & No & No & $\mathrm{Si}$ \\
\hline
\end{tabular}

Tabla 2. Aplicaciones de estereolitografía ${ }^{2}$

\begin{tabular}{ll}
\hline \multicolumn{1}{c}{ Ortodoncia } & \multicolumn{1}{c}{ Cirugía maxilofacial } \\
\hline Diagnóstico y planificación de tratamiento ortodóncico & Simulación de osteotomías en la corrección de asimetrías faciales. \\
Instalacion de microtornillos para anclaje ortodóncico & Demostración de resultados post quirúrgicos en paciente operados de cirugía ortognática. \\
Fabricación de distractor osteogénico & Reconstrucciones en cirugías complejas de traumatismos. \\
Fabricación de aditamentos para tracción de caninos incluídos. & Fabricación de implantes óseos según forma y dimensiones del defecto a restaurar \\
Planificación quirúrgica de caninos incluídos & Colocación precisa de implantes dentales, mediante la construcción de guías quirúrgicas exactas. \\
& Adaptación y/o ubicación de distractor osteogénico antes de la cirugía dependiendo de la necesidad del caso. \\
\hline
\end{tabular}


A pesar de que los equipamientos en la técnica de estereolitografía presenta un alto costo inicial, esta tecnología está cada vez más presente en la práctica odontológica, ya que juega un papel importante en la planificación y ejecución de procedimientos ${ }^{1}$ (Tabla 3 ).

\section{Conclusiones}

Los modelos 3D significan un gran avance para el medio odontológico, siendo la técnica de estereolitografía la más utilizada. Esta técnica debe ser utilizada por el cirujano dentista como un recurso auxiliar, que le puede brindar información específica, sobre todo en aquellos casos de mayor complejidad. De igual manera, existen indicaciones específicas para el uso de la técnica de estereolitografía, evitando así también un costo innecesario.

Las áreas de ortodoncia y cirugía máxilofacial son especialidades que se han visto mayoritariamente favorecidas con la aparición de las técnicas de modelos 3D. Las aplicaciones en estas áreas están siendo cada vez más implementadas debido a la gran cantidad de ventajas de esta tecnología que presenta esta tecnología.

El rol de los modelos obtenidos por estereolitografía se encuentra en continúa actualización, donde cabe mencionar los múltiples usos en diferentes áreas de la medicina, incluso en medicina legal. Esta tecnología permite una mejoría de la comunicación dentro del equipo quirúrgico y con el paciente y, cabe destacar, el aporte en la enseńanza a alumnos gracias a la planificación quirúrgica; mejorando la curva de aprendizaje de éstos.

\section{Referencias Bibliográficas}

1. Alencar P, Roque-Torres G, Meneses-López A, Bóscolo FN, De Almeida S, Groppo FC. Utilización del prototipado rápido en la odontología. Rev Estomatol Herediana. 2015 Abr-Jun;25(2):167-174.

2. Álvarez C, Carrillo J, Fernández J, Grille C. Avances en equipamientos (I): la Estereolitografía y sus materiales, un paso hacia el futuro. Cient Dent. 2006;3(2):151-156.

3. Soares PV, De Almeida Milito G, Pereira FA, Reis BR, Soares CJ, de Sousa Menezes et al. Rapid prototyping and 3D-virtual models for operative dentistry education in Brazil. J Dent Educ. 2013;77(3):358-63.

4. Wu G, Zhou B, Bi Y, Zhao Y. Selective laser sintering technology

\begin{tabular}{ll}
\hline \multicolumn{2}{c}{ Tabla 3. Ventajas y desventajas de la técnica de estereolitografía en odontología ${ }^{2}$} \\
\hline \multicolumn{1}{c}{ Ventajas } & Desventajas \\
Disminuye riesgos quirúrgico. & Alto costo de los equipos. \\
Optimización de tiempo en planificaciones. & Largo tiempo de fabricación de producto final. \\
Mejoría en comunicación odontólogo - paciente. & \\
Simplificación de pasos en técnicas convencionales. &
\end{tabular}

for customized fabrication of facial prostheses. J Prosthet Dent. 2008;100(1):56-60.

5. Anderl H, Zur Nedden D, Mühlbauer W, Twerdy K, Zanon E, Wicke K, et al. CT - Guided stereolithography as a new tool in craniofacial surgery. Br J Plast Surg. 1994;47(1):60-4.

6. Sailer HF, Haers PE, Zollikofer CP, Warnke T, Carls FR, Stucki P. The value of stereolithographic models for preoperative diagnosis of craniofacial deformities and planning of surgical corrections. Int J Oral Maxillofac Surg. 1998;27(5):32733.

7. Salles FA, Anchieta MVM, Carvalho GP. Estereolitografia auxiliando o planejamento cirúrgico em enfermidades orais. Rev Bras Patol Oral. 2002;1(1):54-60.

8. Cunningham JR 1l, Madsen MJ, Peterson G. Stereolithographic modeling technology applied to tumor resection. J Oral Maxillofac Surg. 2005;63(6):873-8.

9. Toro C, Robiony M, Costa F, Zermanand N, Politi M. Feasibility of preoperative planning using anatomical facsimile models for mandibular reconstruction. Head Face Med. 2007;3:5

10. Zinser MJ, Sailer HF, Ritter L, Braumann B, Maegele M, Zoller JE. A paradigm shift in orthognathic surgery? A comparison of navigation, computer-aided designed/computer-aided manufactured splints, and "classic" intermaxillary splints to surgical transfer of virtual orthognathic planning. J Oral Maxillofac Surg. 2013 Dec; 71(12):2151.e1-e21.

11. Morea C, Hayek JE, Oleskovicz C, Dominguez GC, Chilvarquer I. Precise insertion of orthodontic miniscrews with a stereolithographic surgical guide based on cone beam computed tomography data: a pilot study. Int J Oral Maxillofac Implants. 2011;26(4):860- 5.
12. Hazeveld A, Huddleston Slater JJ, Ren Y. Accuracy and reproducibility of dental replica models reconstructed by different rapid prototyping techniques. Am J Orthod Dentofacial Orthop. 2014;145(1):108-15.

13. Cuperus AM, Harms MC, Rangel FA, Bronkhorst EM, ScholsJG, Breuning KH. Dental models made with an intraoral scanner: A validation study. Am J Orthod Dentofacial Orthop. 2012;142:308-13.

14. Choi J, Kim N, Kim Y, Lee J, Kim $\mathrm{M}$, Lee J, et al. Analysis of errors in medical rapid prototyping models. Int J Oral Maxillofac Surg. 2002;31:23-32.

15. Mallepree T, Bergers, D. Accuracy medical RP models. Rapid Prototyping Journal. 2009;5:325-32.

16. El-Katatny I, Masood S, Morsi Y. Error analysis of FDM fabricated medical replicas, Rapid Prototyping Journal. 2010;16:36-43.

17. Germani M, Raffaeli R, Mazzoli A. A method for performance evaluation of RE/RP systems in dentistry. Rapid Prototyping Journal. 2010;16:344-55.

18. Arrieta C, Uribe S, Ramos-Grez J, Vargas A, Irarrazaval P, Parot $\mathrm{V}$, et al. Quantitative assessments of geometric errors for rapid prototyping in medical applications. Rapid Prototyping Journal. 2012;18(6):431-442.

19. Faber J, Berto PM, Quaresma M. Rapid prototyping as a tool for diagnosis and treatment planning for maxillary canine impaction. Am J Orthod Dentofacial Orthop. 2006;129:583-9.

20. Salles F, Anchieta M, Costa Bezerra P, Torres ML, Queiroz E, Faber J. Complete and isolated congenital aglossia: Case report and treatment of sequelae using rapid prototyping models. Oral Surg Oral Med Oral Pathol Oral Radiol Endod. 2008;105e41-47. 